\title{
High breakdown estimators to robustify phase II multivariate control charts.
}

\begin{abstract}
Control chart is a statistical process control tool that is used to monitor the changes in a process. Hotelling's T2 chart is one of the most popular control charts for monitoring independently and identically distributed random vectors. This chart detects many types of out-of-control signals, but it is not sensitive to small shifts in the mean vector. This study propose a more efficient $\mathrm{T} 2$ control charts based on the re-weigted robust estimators of location and dispersion. The proposed control charts are attained by substituting the classical estimators of the mean vector and covariance matrix in the Hotelling's T2 by the re-weighted MCD and re-weighted MVE estimators. In this study, Monte Carlo simulations were carried out to establish the proposed robust control limit. Following that, we suggested suitable estimators for each condition. Our advice in this study is replacing the classical mean vector and covariance matrix of the data in the Hotelling's T2 statistic by there weighted MCD and Re-weighted MVE estimators.
\end{abstract}

Keyword: RMCD estimators; Breakdown point; Hotelling's T2 chart; Outliers; RMVE estimators. 